\title{
Role for creatine metabolism in energy expenditure
}

\section{$C 4$}

...adipose

creatine

metabolism is

a very powerful

component of

diet-induced

thermo-

genesis...

5

To maintain a stable body weight, humans and animals regulate energy expenditure in response to changes in energy intake. The molecular mechanisms that underlie this process are largely unknown; however, new work in mice suggests that creatine metabolism could have a role in the control of energy expenditure during diet-induced obesity.

Previous work had suggested that creatine increased energy expenditure by stimulating ATP

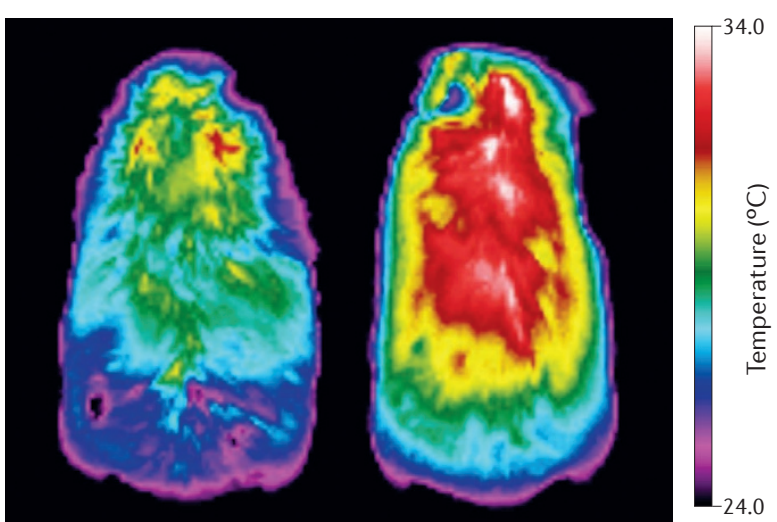

Body temperature of a Gatm ${ }^{\text {flox/flox }}$ mouse (right) and an Adipo-GATM knockout mouse (left) using infrared thermography. Image courtesy of Lawrence Kazak. turnover in the mitochondria, which led Bruce Spiegelman, Lawrence Kazak and colleagues to explore creatine metabolism in adipose tissue and its role in diet-induced thermogenesis. GATM is the rate-limiting enzyme in creatine synthesis, so Gatm ${ }^{\text {flox/flox }}$ mice were crossed with adiponectin-Cre mice to delete Gatm selectively in fat (Adipo-GATM KO). The knockout mice had reduced levels of GATM and exhibited increased cold sensitivity.

Under thermoneutral housing conditions, the knockout mice gained more weight than the flox mice, which indicates that they are prone to obesity. Following highfat feeding, knockout mice also exhibited mild metabolic impairment compared with flox mice. By monitoring food intake and resting metabolic rate, the researchers were able to show that the knockout mice had impaired energy expenditure rather than increased energy intake. Adaptive thermogenesis was also demonstrated to be impaired in Adipo-GATM KO mice, as the metabolic response to both adrenergic stimulation and caloric excess was severely blunted compared with control mice. Importantly, creatine supplementation rescued many of the adverse effects seen in the Adipo-GATM KO mice.

"These studies show that adipose creatine metabolism is a very powerful component of diet-induced thermogenesis and resistance to obesity," explains Spiegelman. However, as this study was conducted in mice, more work needs to be done to determine the role of creatine metabolism in humans. "We would like to see how this pathway can be used in humans to influence and improve metabolic control and resistance to obesity and diabetes mellitus," says Spiegelman. "We also want to complete our understanding of all of the steps in the pathway that are not yet completely understood."

\section{Claire Greenhill}

ORIGINAL ARTICLE Kazak, L. et al. Genetic depletion of adipocyte creatine metabolism inhibits diet-induced thermogenesis and drives obesity. Cell Metab. http://dx.doi.org/10.1016/j. cmet.2017.08.009 (2017) 